\title{
IAU Joint Discussion 9: Are the Fundamental Constants Varying in Space-time?
}

\author{
Paolo Molaro $^{1}$ and Elisabeth Vangioni ${ }^{2}$ \\ ${ }^{1}$ INAF -Osservatorio Astronomico di Trieste, Via Tiepolo 11, I-34131 Trieste, Italy \\ ${ }^{2}$ Institut d' Astrophysique de Paris, 98bis, bd Arago, F-75014 Paris, France
}

The Joint Discussion on the variability of fundamental constants within the IAU GA 2009 was organized with the coordination of the IAU Division VIII Galaxies \& the Universe and the support of IAU Commissions N. 47 Cosmology, N. 52 Relativity in Fundamental Astronomy, N.40 Radio Astronomy and N. 30 Radial velocities. The JD provided a timely opportunity to confront different points of view on this rather exciting subject.

The fundamental constants determine the scales of natural phenomena and many theories beyond the standard model of physics predict their variation. Such a possibility occupies quite a prominent place in cosmology since the existence of scalar fields, of the kind invoked to explain the universal acceleration, coupling with electromagnetic field or ordinary matter might lead to dynamic constants. The study of the behaviour of these quantities throughout the history of the universe is an effective way to probe fundamental physics and this is why on investigation of this subject is highly recommended in the Science Vision Document, in the ESA-ESO Working Group report on Fundamental Cosmology, and is one of the science drivers for the next facilities such as the E-ELT

High precision frequency measurements with atomic clocks in the laboratories established the fine structure constant to 17 significant figures. However, the variation is likely non linear in time and only astronomical observations can tell how they have been varying back in time. The Oklo natural reactor allows us to go back in time by about 2 Gyrs and meteorites to the birth of the solar system, QSO absorption systems bring us to about $80 \%$ of the age of the Universe while $\mathrm{CMB}$ and BBN to even earlier epochs. In 2001, observations of spectral lines in distant QSOs brought the first hints that the fine structure constant might change its value over time, with a variation of few parts per million. However, the subject is presently controversial with some other evidence which suggests null variation. The most active researchers in this area attended the Joint Discussion and discussed the latest developments. The issue was lively debated but a clear solution remains still to be found.

We wish to thank all participants to the meeting for their enthusiasm and for sharing their most recent results. We wish to thank the Scientific Organizing Committee for its advice and help in organizing the scientific program. The SOC was composed of

John D. Barrow, Francoise Combes, Thomas Dent, Sandro D'Odorico, Victor Flambaum, Sergei Levshakov, Carlos Martins, Michael Murphy, Cedric Ledoux, Keith Olive, Patrick Petitjean, Dieter Reimers, Raghunathan Srianand, Jean-Philippe Uzan, John Webb.

The Brazilian Astronomical society provided an efficient local organization and is also warmly thanked. 